\title{
DIRECT IMAGES OF BUNDLES UNDER FROBENIUS MORPHISM
}

\author{
XIAOTAO SUN
}

\begin{abstract}
Let $X$ be a smooth projective variety of dimension $n$ over an algebraically closed field $k$ with $\operatorname{char}(k)=p>0$ and $F: X \rightarrow X_{1}$ be the relative Frobenius morphism. For any vector bundle $W$ on $X$, we prove that instability of $F_{*} W$ is bounded by instability of $W \otimes \mathrm{T}^{\ell}\left(\Omega_{X}^{1}\right)(0 \leq \ell \leq n(p-1))$ (Corollary 4.9). When $X$ is a smooth projective curve of genus $g \geq 2$, it implies $F_{*} W$ being stable whenever $W$ is stable.
\end{abstract}

Dedicated to Professor Zhexian Wan on the occasion of his 80th birthday.

\section{INTRODUCTION}

Let $X$ be a smooth projective variety of dimension $n$ over an algebraically closed field $k$ with $\operatorname{char}(k)=p>0$. Fix an ample divisor $\mathrm{H}$ on $X$, by a semistable (resp. stable) torsion free sheaf, we mean a H-slope semistable (resp. H-slope stable) sheaf in this paper. For a torsion free sheaf $\mathcal{F}$ on $X$, there is a unique filtration

$$
0=\mathcal{F}_{0} \subset \mathcal{F}_{1} \subset \cdots \subset \mathcal{F}_{k}=\mathcal{F}
$$

such that $\mathcal{F}_{i} / \mathcal{F}_{i-1}(1 \leq i \leq k)$ are semistable torsion free sheaves and

$$
\mu_{\max }(\mathcal{F}):=\mu\left(\mathcal{F}_{1}\right)>\mu\left(\mathcal{F}_{2} / \mathcal{F}_{1}\right)>\cdots>\mu\left(\mathcal{F}_{k} / \mathcal{F}_{k-1}\right):=\mu_{\min }(\mathcal{F}) .
$$

The instability of $\mathcal{F}$ was defined as $\mathrm{I}(\mathcal{F})=\mu_{\max }(\mathcal{F})-\mu_{\min }(\mathcal{F})$, which measures how far from $\mathcal{F}$ being semi-stable. In particular, $\mathcal{F}$ is semistable if and only if $\mathrm{I}(\mathcal{F})=0$. On the other hand, there are sub-bundles $\mathrm{T}^{\ell}\left(\Omega_{X}^{1}\right) \subset\left(\Omega_{X}^{1}\right)^{\otimes \ell}, 0 \leq \ell \leq n(p-1)$, which are the associated bundles of $\Omega_{X}^{1}$ through some elementary (perhaps interesting) representations of $\mathrm{GL}(n)$. These representations do not appear in characteristic zero.

Let $F: X \rightarrow X_{1}$ be the relative Frobenius morphism, for any vector bundle $W$ on $X$, let $\mathrm{I}(W, X)$ be the maximal value of $\mathrm{I}\left(W \otimes \mathrm{T}^{\ell}\left(\Omega_{X}^{1}\right)\right)$

Date: February 22, 2008.

Partially supported by a matched grant of Chinese Academy of Science and the DFG Leibniz Preis of Esnault-Viehweg. 
where $0 \leq \ell \leq n(p-1)$. Then one of our results in this paper shows (Corollary 4.9): When $K_{X} \cdot \mathrm{H}^{n-1} \geq 0$, we have

$$
\mathrm{I}\left(F_{*} W\right) \leq p^{n-1} \operatorname{rk}(W) \mathrm{I}(W, X) .
$$

In particular, if the bundles $W \otimes \mathrm{T}^{\ell}\left(\Omega_{X}^{1}\right), 0 \leq \ell \leq n(p-1)$, are semistable, then $F_{*} W$ is semistable. In fact, when $K_{X} \cdot \mathrm{H}^{n-1}>0$, we can show that the stability of $W \otimes \mathrm{T}^{\ell}\left(\Omega_{X}^{1}\right), 0 \leq \ell \leq n(p-1)$, implies the stability of $F_{*} W$ (Theorem 4.8).

The main theorem has an immediate corollary that when $X$ is a smooth projective curve of genus $g \geq 2$, the stability of $W$ implies stability of $F_{*} W$. This is in fact our original motivation stimulated by a question raised by Herbert Lange at a conference. When $W$ is a line bundle, it is due to Lange and Pauly ([6, Proposition 1.2$]$ ). The present version is based on our earlier preprint ([8]), where the theorem was completely proved only for curves. It should be pointed out, in case of curves, Mehta and Pauly have proved independently that semi-stability of $W$ implies semi-stability of $F_{*} W$ by a different method. However, their method was not able to prove the stability of $F_{*} W$ when $W$ is stable. In fact, they asked the question: Is stability also preserved by $F_{*}$ ? (cf. [7, Section 7] for the discussions).

To describe the idea of proof, let us compare it to its opposite case, a Galois étale $G$-cover $f: Y \rightarrow X$. Recall that for a semi-stable bundle $W$ on $Y$, to prove semistability of $f_{*} W$, one uses the fact that $f^{*}\left(f_{*} W\right)$ decomposes into pieces of $W^{\sigma}(\sigma \in G)$. To imitate this idea for $F: X \rightarrow X_{1}$, we need a similar decomposition of $V=F^{*}\left(F_{*} W\right)$. Indeed, use the canonical connection $\nabla: V \rightarrow V \otimes \Omega_{X}^{1}$, Joshi-RamananXia-Yu have defined in [4] for $\operatorname{dim}(X)=1$ a canonical filtration

$$
0=V_{p} \subset V_{p-1} \subset \cdots \subset V_{\ell} \subset V_{\ell-1} \subset \cdots V_{1} \subset V_{0}=V
$$

such that $V_{\ell} / V_{\ell+1} \cong W \otimes\left(\Omega_{X}^{1}\right)^{\otimes \ell}$. It is this filtration and its generalization that we are going to use for the study of $F_{*} W$.

As the first step, we generalize the canonical filtration to higher dimensional $X$. Its definition can be generalized straightforwardly by using the canonical connection $\nabla: V \rightarrow V \otimes \Omega_{X}^{1}$. The study of its graded quotients are much involved. We show (Theorem 3.7) that there exists a canonical filtration

$$
0=V_{n(p-1)+1} \subset V_{n(p-1)} \subset \cdots \subset V_{1} \subset V_{0}=V=F^{*}\left(F_{*} W\right)
$$

such that $\nabla$ induces injective morphisms $V_{\ell} / V_{\ell+1} \stackrel{\nabla}{\rightarrow}\left(V_{\ell-1} / V_{\ell}\right) \otimes \Omega_{X}^{1}$ of vector bundles and the isomorphisms $V_{\ell} / V_{\ell+1} \cong W \otimes \mathrm{T}^{\ell}\left(\Omega_{X}^{1}\right)$, where $\mathrm{T}^{\ell}\left(\Omega_{X}^{1}\right) \subset\left(\Omega_{X}^{1}\right)^{\otimes \ell}$ are subbundles given by representations of $\operatorname{GL}(n)$ (cf. Definition 3.4). In characteristic zero, $\mathrm{T}^{\ell}\left(\Omega_{X}^{1}\right)=\operatorname{Sym}^{\ell}\left(\Omega_{X}^{1}\right)$. In 
characteristic $p>0, \mathrm{~T}^{\ell}\left(\Omega_{X}^{1}\right) \cong \operatorname{Sym}^{\ell}\left(\Omega_{X}^{1}\right)$ only for $\ell<p$. In general, there is a resolution of $\mathrm{T}^{\ell}\left(\Omega_{X}^{1}\right)$ (Proposition 3.5) by symmetric powers of $\Omega_{X}^{1}$ and exterior powers of $F^{*}\left(\Omega_{X}^{1}\right)$ (After [8] appeared, Indranil Biswas told me that a similar filtration was defined and studied in Proposition 4.1 of their preprint [1]. However, since their map (4.7) was wrong, the Proposition 4.1 (also Proposition 4.2 consequently) of 11 was wrong. After we pointed out these gaps, they have corrected these mistakes in [2]).

To prove the main theorem, we also need to compare sub-sheaves of $V_{\ell} / V_{\ell+1}$ to sub-sheaves of $V_{n(p-1)-\ell} / V_{n(p-1)-\ell+1}$ which are $\nabla$-invariant (Proposition 4.7). It is reduced to consider the (graded) $K$-algebra

$$
R=\frac{K\left[y_{1}, y_{2}, \cdots, y_{n}\right]}{\left(y_{1}^{p}, y_{2}^{p}, \ldots, y_{n}^{p}\right)}=\bigoplus_{\ell=0}^{n(p-1)} R^{\ell}
$$

with a D-module structure, where

$$
\mathrm{D}=\frac{K\left[\partial_{y_{1}}, \cdots, \partial_{y_{n}}\right]}{\left(\partial_{y_{1}}^{p}, \cdots, \partial_{y_{n}}^{p}\right)}=K\left[t_{1}, t_{2}, \cdots, t_{n}\right]=\bigoplus_{\ell=0}^{n(p-1)} \mathrm{D}_{\ell}
$$

which acts on $R$ through the partial derivations $\partial_{y_{1}}, \partial_{y_{2}}, \ldots, \partial_{y_{n}}$. For any subspace $V \subset R^{\ell}$, let $\mathbb{L}\left(\mathrm{D}_{2 \ell-n(p-1)} \cdot V\right)$ be the linear subspace spanned by $\mathrm{D}_{2 \ell-n(p-1)} \cdot V \subset R^{n(p-1)-\ell}$. Then we are reduced to ask if

$$
\operatorname{dim}(V) \leq \operatorname{dim} \mathbb{L}\left(\mathrm{D}_{2 \ell-n(p-1)} \cdot V\right) \quad \text { when } \frac{n(p-1)}{2} \leq \ell \leq n(p-1) ?
$$

Our Lemma 4.5 and Proposition 4.7 give an affrmative answer to it.

When $X$ is a smooth projective curve of genus $g \geq 1$, the proof of theorem is very elementary and simple, which does not need the more involved arguments of higher dimensional case and shows the idea of proof best. Thus, although it is a direct corollary of the general case (Theorem 4.8), we still put its proof in an independent section. It is also convenient for a reader who is only interested in the proof for curves.

Acknowledgements: I would like to thank Hélène Esnault, Eckart Viehweg, Hourong Qin, Manfred Lehn, Indranil Biswas, Herbert Lange, Christian Pauly for their interest and discussions. The proof of the purely combinatorial Lemma 4.6 is due to Fusheng Leng. I thank him very much for his help. Finally, I would like to thank the referee for the helpful comments. 


\section{The CASE OF CURVES}

Let $k$ be an algebraically closed field of characteristic $p>0$ and $X$ be a smooth projective curve over $k$. Let $F: X \rightarrow X_{1}$ be the relative $k$-linear Frobenius morphism, where $X_{1}:=X \times_{k} k$ is the base change of $X / k$ under the Frobenius $\operatorname{Spec}(k) \rightarrow \operatorname{Spec}(k)$. Let $W$ be a vector bundle on $X$ and $V=F^{*}\left(F_{*} W\right)$. It is known ([5, Theorem 5.1]) that $V$ has a canonical connection $\nabla: V \rightarrow V \otimes \Omega_{X}^{1}$ with zero $p$-curvature. In [4, Section 5], the authors defined a canonical filtration

$$
0=V_{p} \subset V_{p-1} \subset \cdots \subset V_{\ell} \subset V_{\ell-1} \subset \cdots V_{1} \subset V_{0}=V
$$

where $V_{1}=\operatorname{ker}\left(V=F^{*} F_{*} W \rightarrow W\right)$ and

$$
V_{\ell+1}=\operatorname{ker}\left(V_{\ell} \stackrel{\nabla}{\rightarrow} V \otimes \Omega_{X}^{1} \rightarrow V / V_{\ell} \otimes \Omega_{X}^{1}\right) .
$$

The following lemma belongs to them (cf. [4, Theorem 5.3]).

Lemma 2.1. $\quad$ (i) $V_{0} / V_{1} \cong W, \nabla\left(V_{\ell+1}\right) \subset V_{\ell} \otimes \Omega_{X}^{1}$ for $\ell \geq 1$.

(ii) $V_{\ell} / V_{\ell+1} \stackrel{\nabla}{\rightarrow}\left(V_{\ell-1} / V_{\ell}\right) \otimes \Omega_{X}^{1}$ is an isomorphism for $1 \leq \ell \leq p-1$.

(iii) If $g \geq 2$ and $W$ is semistable, then the canonical filtration (2.1) is nothing but the Harder-Narasimhan filtration.

Proof. (i) follows by the definition, which and (ii) imply (iii). To prove (ii), let $I_{0}=F^{*} F_{*} \mathcal{O}_{X}, I_{1}=\operatorname{ker}\left(F^{*} F_{*} \mathcal{O}_{X} \rightarrow \mathcal{O}_{X}\right)$ and

$$
I_{\ell+1}=\operatorname{ker}\left(I_{\ell} \stackrel{\nabla}{\rightarrow} I_{0} \otimes \Omega_{X}^{1} \rightarrow I_{0} / I_{\ell} \otimes \Omega_{X}^{1}\right)
$$

which is the canonical filtration (2.1) in the case $W=\mathcal{O}_{X}$.

(ii) is clearly a local problem, we can assume $X=\operatorname{Spec}(k[[x]])$ and $W=k[[x]]^{\oplus r}$. Then $V_{0}:=V=F^{*}\left(F_{*} W\right)=I_{0}^{\oplus r}, V_{\ell}=I_{\ell}^{\oplus r}$ and

$$
V_{\ell} / V_{\ell+1}=\left(I_{\ell} / I_{\ell+1}\right)^{\oplus r} \stackrel{\oplus \nabla}{\longrightarrow}\left(I_{\ell-1} / I_{\ell} \otimes \Omega_{X}^{1}\right)^{\oplus r}=V_{\ell-1} / V_{\ell} \otimes \Omega_{X}^{1} .
$$

Thus it is enough to show that

$$
I_{\ell} / I_{\ell+1} \stackrel{\nabla}{\rightarrow} I_{\ell-1} / I_{\ell} \otimes \Omega_{X}^{1}
$$

is an isomorphism. Locally, $I_{0}=k[[x]] \otimes_{k\left[\left[x^{p}\right]\right]} k[[x]]$ and

$$
\nabla: k[[x]] \otimes_{k\left[\left[x^{p}\right]\right]} k[[x]] \rightarrow I_{0} \otimes_{\mathcal{O}_{X}} \Omega_{X}^{1},
$$

where $\nabla(g \otimes f)=g \otimes f^{\prime} \otimes \mathrm{d} x$. The $\mathcal{O}_{X^{-}}$module

$$
I_{1}:=\operatorname{ker}\left(k[[x]] \otimes_{k\left[\left[x^{p}\right]\right]} k[[x]] \rightarrow k[[x]]\right)
$$

has a basis $\left\{x^{i} \otimes 1-1 \otimes x^{i}\right\}_{1 \leq i \leq p-1}$. Notice that $I_{1}$ is also an ideal of the $\mathcal{O}_{X^{-}}$algebra $I_{0}=k[[x]] \otimes_{k\left[\left[x^{p}\right]\right]} k[[x]]$, let $\alpha=x \otimes 1-1 \otimes x$, then $\alpha^{i} \in I_{1}$. 
It is easy to see that $\alpha, \alpha^{2}, \ldots, \alpha^{p-1}$ is a basis of the $\mathcal{O}_{X}$-module $I_{1}$ (notice that $\alpha^{p}=x^{p} \otimes 1-1 \otimes x^{p}=0$ ), and

$$
\nabla\left(\alpha^{\ell}\right)=-\ell \alpha^{\ell-1} \otimes \mathrm{d} x .
$$

Thus, as a free $\mathcal{O}_{X}$-module, $I_{\ell}$ has a basis $\left\{\alpha^{\ell}, \alpha^{\ell+1}, \ldots, \alpha^{p-1}\right\}$, which means that $I_{\ell} / I_{\ell+1}$ has a basis $\alpha^{\ell},\left(I_{\ell-1} / I_{\ell}\right) \otimes \Omega_{X}^{1}$ has a basis $\alpha^{\ell-1} \otimes \mathrm{d} x$ and $\nabla\left(\alpha^{\ell}\right)=-\ell \alpha^{\ell-1} \otimes \mathrm{d} x$. Therefore $\nabla$ induces the isomorphism (2.5) since $(\ell, p)=1$, which implies the isomorphism in (ii).

Theorem 2.2. Let $X$ be a smooth projective curve of genus $g \geq 1$. Then $F_{*} W$ is semi-stable whenever $W$ is semi-stable. If $g \geq 2$, then $F_{*} W$ is stable whenever $W$ is stable.

Proof. Let $\mathcal{E} \subset F_{*} W$ be a nontrivial subbundle and

$$
0 \subset V_{m} \cap F^{*} \mathcal{E} \subset \cdots \subset V_{1} \cap F^{*} \mathcal{E} \subset V_{0} \cap F^{*} \mathcal{E}=F^{*} \mathcal{E}
$$

be the induced filtration. Let $r_{\ell}=\operatorname{rk}\left(\frac{V_{\ell} \cap F^{*} \mathcal{E}}{V_{\ell+1} \cap F^{*} \mathcal{E}}\right)$ be the ranks of quotients. Then, by the filtration (2.9), we have

$$
\mu\left(F^{*} \mathcal{E}\right)=\frac{1}{\operatorname{rk}\left(F^{*} \mathcal{E}\right)} \sum_{\ell=0}^{m} r_{\ell} \cdot \mu\left(\frac{V_{\ell} \cap F^{*} \mathcal{E}}{V_{\ell+1} \cap F^{*} \mathcal{E}}\right) .
$$

By Lemma 2.1, $V_{\ell} / V_{\ell+1} \cong W \otimes\left(\Omega_{X}^{1}\right)^{\otimes \ell}$ is stable, we have

$$
\mu\left(\frac{V_{\ell} \cap F^{*} \mathcal{E}}{V_{\ell+1} \cap F^{*} \mathcal{E}}\right) \leq \mu(W)+2(g-1) \ell .
$$

Then, notice that $\mu(V)=\mu(W)+(p-1)(g-1)$, we have

$$
\mu\left(F_{*} W\right)-\mu(\mathcal{E}) \geq \frac{2 g-2}{p \cdot \operatorname{rk}(\mathcal{E})} \cdot \sum_{\ell=0}^{m}\left(\frac{p-1}{2}-\ell\right) r_{\ell}
$$

which becomes equality if and only if the inequalities in (2.11) become equalities. It is clear by (2.12) that $\mu\left(F_{*} W\right)-\mu(\mathcal{E})>0$ if $m \leq \frac{p-1}{2}$. Thus we can assume that $m>\frac{p-1}{2}$, then we can write

$$
\begin{gathered}
\sum_{\ell=0}^{m}\left(\frac{p-1}{2}-\ell\right) r_{\ell}=\sum_{\ell=m+1}^{p-1}\left(\ell-\frac{p-1}{2}\right) r_{p-1-\ell} \\
\quad+\sum_{\ell>\frac{p-1}{2}}^{m}\left(\ell-\frac{p-1}{2}\right)\left(r_{p-1-\ell}-r_{\ell}\right) \\
\geq \sum_{\ell>\frac{p-1}{2}}^{m}\left(\ell-\frac{p-1}{2}\right)\left(r_{p-1-\ell}-r_{\ell}\right) .
\end{gathered}
$$


On the other hand, since the isomorphisms $V_{\ell} / V_{\ell+1} \stackrel{\nabla}{\rightarrow}\left(V_{\ell-1} / V_{\ell}\right) \otimes \Omega_{X}^{1}$ in Lemma 2.1 (ii) induce the injections

$$
\frac{V_{\ell} \cap F^{*} \mathcal{E}}{V_{\ell+1} \cap F^{*} \mathcal{E}} \hookrightarrow \frac{V_{\ell-1} \cap F^{*} \mathcal{E}}{V_{\ell} \cap F^{*} \mathcal{E}} \otimes \Omega_{X}^{1}
$$

we have $r_{0} \geq r_{1} \geq \cdots \geq r_{\ell-1} \geq r_{\ell} \geq \cdots \geq r_{m}$. Thus

$$
\mu\left(F_{*} W\right)-\mu(\mathcal{E}) \geq \frac{2 g-2}{p \cdot \operatorname{rk}(\mathcal{E})} \sum_{\ell=0}^{m}\left(\frac{p-1}{2}-\ell\right) r_{\ell} \geq 0 .
$$

If $\mu\left(F_{*} W\right)-\mu(\mathcal{E})=0$, then (2.12) and (2.13) become equalities. That (2.12) becomes equality implies inequalities in (2.11) become equalities, which means $r_{0}=r_{1}=\cdots=r_{m}=\operatorname{rk}(W)$. Then that (2.13) become equalities implies $m=p-1$. Altogether imply $\mathcal{E}=F_{*} W$, we get contradiction. Hence $F_{*} W$ is stable whenever $W$ is stable.

\section{THE FILTRATION ON HIGHER DIMENSION VARIETIES}

Let $X$ be a smooth projective variety over $k$ of dimension $n$ and $F: X \rightarrow X_{1}$ be the relative $k$-linear Frobenius morphism, where $X_{1}:=$ $X \times_{k} k$ is the base change of $X / k$ under the Frobenius $\operatorname{Spec}(k) \rightarrow$ Spec $(k)$. Let $W$ be a vector bundle on $X$ and $V=F^{*}\left(F_{*} W\right)$. We have the straightforward generalization of the canonical filtration to higher dimensional varieties.

Definition 3.1. Let $V_{0}:=V=F^{*}\left(F_{*} W\right), V_{1}=\operatorname{ker}\left(F^{*}\left(F_{*} W\right) \rightarrow W\right)$

$$
V_{\ell+1}:=\operatorname{ker}\left(V_{\ell} \stackrel{\nabla}{\rightarrow} V \otimes_{\mathcal{O}_{X}} \Omega_{X}^{1} \rightarrow\left(V / V_{\ell}\right) \otimes_{\mathcal{O}_{X}} \Omega_{X}^{1}\right)
$$

where $\nabla: V \rightarrow V \otimes_{\mathcal{O}_{X}} \Omega_{X}^{1}$ is the canonical connection (cf. [5, Theorem $5.1])$.

We first consider the special case $W=\mathcal{O}_{X}$ and give some local descriptions. Let $I_{0}=F^{*}\left(F_{*} \mathcal{O}_{X}\right), I_{1}=\operatorname{ker}\left(F^{*} F_{*} \mathcal{O}_{X} \rightarrow \mathcal{O}_{X}\right)$ and

$$
I_{\ell+1}=\operatorname{ker}\left(I_{\ell} \stackrel{\nabla}{\rightarrow} I_{0} \otimes_{\mathcal{O}_{X}} \Omega_{X}^{1} \rightarrow I_{0} / I_{\ell} \otimes_{\mathcal{O}_{X}} \Omega_{X}^{1}\right) .
$$

Locally, let $X=\operatorname{Spec}(A), I_{0}=A \otimes_{A^{p}} A$, where $A=k\left[\left[x_{1}, \cdots, x_{n}\right]\right]$, $A^{p}=k\left[\left[x_{1}^{p}, \cdots, x_{n}^{p}\right]\right]$. Then the canonical connection $\nabla: I_{0} \rightarrow I_{0} \otimes \Omega_{X}^{1}$ is locally defined by

$$
\nabla\left(g \otimes_{A^{p}} f\right)=\sum_{i=1}^{n}\left(g \otimes_{A^{p}} \frac{\partial f}{\partial x_{i}}\right) \otimes_{A} \mathrm{~d} x_{i}
$$


Notice that $I_{0}$ has an $A$-algebra structure such that $I_{0}=A \otimes_{A^{p}} A \rightarrow A$ is a homomorphism of $A$-algebras, its kernel $I_{1}$ contains elements

$$
\alpha_{1}^{k_{1}} \alpha_{2}^{k_{2}} \cdots \alpha_{n}^{k_{n}}, \text { where } \alpha_{i}=x_{i} \otimes_{A^{p}} 1-1 \otimes_{A^{p}} x_{i}, \quad \sum_{i=1}^{n} k_{i} \geq 1 .
$$

Since $\alpha_{i}^{p}=x_{i}^{p} \otimes_{A^{p}} 1-1 \otimes_{A^{p}} x_{i}^{p}=0$, the set $\left\{\alpha_{1}^{k_{1}} \cdots \alpha_{n}^{k_{n}} \mid k_{1}+\cdots+k_{n} \geq 1\right\}$ has $p^{n}-1$ elements. In fact, we have

Lemma 3.2. Locally, as free A-modules, we have, for all $\ell \geq 1$,

$$
I_{\ell}=\bigoplus_{k_{1}+\cdots+k_{n} \geq \ell}\left(\alpha_{1}^{k_{1}} \cdots \alpha_{n}^{k_{n}}\right) A
$$

Proof. We first prove for $\ell=1$ that $\left\{\alpha_{1}^{k_{1}} \cdots \alpha_{n}^{k_{n}} \mid k_{1}+\cdots+k_{n} \geq 1\right\}$ is a basis of $I_{1}$ locally. By definition, $I_{1}$ is locally free of rank $p^{n}-1$, thus it is enough to show that as an $A$-module $I_{1}$ is generated locally by $\left\{\alpha_{1}^{k_{1}} \cdots \alpha_{n}^{k_{n}} \mid k_{1}+\cdots+k_{n} \geq 1\right\}$ since it has exactly $p^{n}-1$ elements.

It is easy to see that as an $A$-module $I_{1}$ is locally generated by $\left\{x_{1}^{k_{1}} \cdots x_{n}^{k_{n}} \otimes_{A^{p}} 1-1 \otimes_{A^{p}} x_{1}^{k_{1}} \cdots x_{n}^{k_{n}} \mid k_{1}+\cdots+k_{n} \geq 1,0 \leq k_{i} \leq p-1\right\}$.

It is enough to show that any $x_{1}^{k_{1}} \cdots x_{n}^{k_{n}} \otimes_{A^{p}} 1-1 \otimes_{A^{p}} x_{1}^{k_{1}} \cdots x_{n}^{k_{n}}$ is a linear combination of $\left\{\alpha_{1}^{k_{1}} \cdots \alpha_{n}^{k_{n}} \mid k_{1}+\cdots+k_{n} \geq 1\right\}$. The claim is obvious when $k_{1}+\cdots+k_{n}=1$, we consider the case $k_{1}+\cdots+k_{n}>1$. Without loss generality, assume $k_{n} \geq 1$ and there are $f_{j_{1}, \ldots, j_{n}} \in A$ such that

$$
x_{1}^{k_{1}} \cdots x_{n}^{k_{n}-1} \otimes_{A^{p}} 1-1 \otimes_{A^{p}} x_{1}^{k_{1}} \cdots x_{n}^{k_{n}-1}=\sum_{j_{1}+\cdots+j_{n} \geq 1}\left(\alpha_{1}^{j_{1}} \cdots \alpha_{n}^{j_{n}}\right) \cdot f_{j_{1}, \ldots, j_{n}} .
$$

Then we have

$$
\begin{aligned}
& x_{1}^{k_{1}} \cdots x_{n}^{k_{n}} \otimes_{A^{p}} 1-1 \otimes_{A^{p}} x_{1}^{k_{1}} \cdots x_{n}^{k_{n}}=\sum_{j_{1}+\cdots+j_{n} \geq 1}\left(\alpha_{1}^{j_{1}} \cdots \alpha_{n}^{j_{n}+1}\right) \cdot f_{j_{1}, \ldots, j_{n}} \\
& +\sum_{j_{1}+\cdots+j_{n} \geq 1}\left(\alpha_{1}^{j_{1}} \cdots \alpha_{n}^{j_{n}}\right) \cdot f_{j_{1}, \ldots, j_{n}} x_{n}+\alpha_{n} \cdot\left(x_{1}^{k_{1}} \cdots x_{n}^{k_{n}-1}\right) .
\end{aligned}
$$

For $\ell>1$, to prove the lemma, we first show

$$
\nabla\left(\alpha_{1}^{k_{1}} \cdots \alpha_{n}^{k_{n}}\right)=-\sum_{i=1}^{n} k_{i}\left(\alpha_{1}^{k_{1}} \cdots \alpha_{i}^{k_{i}-1} \cdots \alpha_{n}^{k_{n}}\right) \otimes_{A} \mathrm{~d} x_{i}
$$

Indeed, (3.6) is true when $k_{1}+\cdots+k_{n}=1$. If $k_{1}+\cdots+k_{n}>1$, we assume $k_{n} \geq 1$ and $\alpha_{1}^{k_{1}} \cdots \alpha_{n}^{k_{n}-1}=\sum g_{j} \otimes_{A^{p}} f_{j}$. Then

$$
\alpha_{1}^{k_{1}} \cdots \alpha_{n}^{k_{n}}=\sum_{j} x_{n} g_{j} \otimes_{A^{p}} f_{j}-\sum_{j} g_{j} \otimes_{A^{p}} f_{j} x_{n} .
$$


Use (3.3), straightforward computations show

$$
\nabla\left(\alpha_{1}^{k_{1}} \cdots \alpha_{n}^{k_{n}}\right)=\alpha_{n} \nabla\left(\alpha_{1}^{k_{1}} \cdots \alpha_{n}^{k_{n}-1}\right)-\left(\alpha_{1}^{k_{1}} \cdots \alpha_{n}^{k_{n}-1}\right) \otimes_{A} \mathrm{~d} x_{n}
$$

which implies (3.6). Now we can assume the lemma is true for $I_{\ell-1}$ and recall that $I_{\ell}=\operatorname{ker}\left(I_{\ell-1} \stackrel{\nabla}{\rightarrow} I_{0} \otimes_{A} \Omega_{X}^{1} \rightarrow\left(I_{0} / I_{\ell-1}\right) \otimes_{A} \Omega_{X}^{1}\right)$. For any

$$
\beta=\sum_{k_{1}+\cdots k_{n} \geq \ell-1}\left(\alpha_{1}^{k_{1}} \cdots \alpha_{n}^{k_{n}}\right) \cdot f_{k_{1}, \ldots, k_{n}} \in I_{\ell-1}, \quad f_{k_{1}, \ldots, k_{n}} \in A,
$$

by using (3.6), we see that $\beta \in I_{\ell}$ if and only if

$$
\sum_{k_{1}+\cdots+k_{n}=\ell-1}\left(\alpha_{1}^{k_{1}} \cdots \alpha_{j}^{k_{j}-1} \cdots \alpha_{n}^{k_{n}}\right) \cdot k_{j} f_{k_{1}, \ldots, k_{n}} \in I_{\ell-1}
$$

for all $1 \leq j \leq n$. Since $\left\{\alpha_{1}^{k_{1}} \cdots \alpha_{n}^{k_{n}} \mid k_{1}+\cdots+k_{n} \geq 1\right\}$ is a basis of $I_{1}$ locally and the lemma is true for $I_{\ell-1}$, (3.7) is equivalent to

For given $\left(k_{1}, \ldots, k_{n}\right)$ with $k_{1}+\cdots+k_{n}=\ell-1$

$$
k_{j} f_{k_{1}, \ldots, k_{n}}=0 \text { for all } j=1, \ldots, n
$$

which implies $f_{k_{1}, \ldots, k_{n}}=0$ whenever $k_{1}+\cdots+k_{n}=\ell-1$. Thus $I_{\ell}$ is generated by $\left\{\alpha_{1}^{k_{1}} \cdots \alpha_{n}^{k_{n}} \mid k_{1}+\cdots+k_{n} \geq \ell\right\}$.

Lemma 3.3. $\quad$ (i) $I_{\ell}=0$ when $\ell>n(p-1)$, and $\nabla\left(I_{\ell+1}\right) \subset I_{\ell} \otimes \Omega_{X}^{1}$ for $\ell \geq 1$.

(ii) $I_{\ell} / I_{\ell+1} \stackrel{\nabla}{\rightarrow}\left(I_{\ell-1} / I_{\ell}\right) \otimes \Omega_{X}^{1}$ are injective in the category of vector bundles for $1 \leq \ell \leq n(p-1)$. In particular, their composition

$$
\nabla^{\ell}: I_{\ell} / I_{\ell+1} \rightarrow\left(I_{0} / I_{1}\right) \otimes_{\mathcal{O}_{X}}\left(\Omega_{X}^{1}\right)^{\otimes \ell}=\left(\Omega_{X}^{1}\right)^{\otimes \ell}
$$

is injective in the category of vector bundles.

Proof. (i) follows from Lemma 3.2 and Definition 3.1. (ii) follows from (3.6).

In order to describe the image of $\nabla^{\ell}$ in (3.9), we recall a GL(n)representation $\mathrm{T}^{\ell}(V) \subset V^{\otimes \ell}$ where $V$ is the standard representation of $\mathrm{GL}(n)$. Let $\mathrm{S}_{\ell}$ be the symmetric group of $\ell$ elements with the action on $V^{\otimes \ell}$ by $\left(v_{1} \otimes \cdots \otimes v_{\ell}\right) \cdot \sigma=v_{\sigma(1)} \otimes \cdots \otimes v_{\sigma(\ell)}$ for $v_{i} \in V$ and $\sigma \in \mathrm{S}_{\ell}$. Let $e_{1}, \ldots, e_{n}$ be a basis of $V$, for $k_{i} \geq 0$ with $k_{1}+\cdots+k_{n}=\ell$ define

$$
v\left(k_{1}, \ldots, k_{n}\right)=\sum_{\sigma \in \mathrm{S}_{\ell}}\left(e_{1}^{\otimes k_{1}} \otimes \cdots \otimes e_{n}^{\otimes k_{n}}\right) \cdot \sigma
$$

Definition 3.4. Let $\mathrm{T}^{\ell}(V) \subset V^{\otimes \ell}$ be the linear subspace generated by all vectors $v\left(k_{1}, \ldots, k_{n}\right)$ for all $k_{i} \geq 0$ satisfying $k_{1}+\cdots+k_{n}=\ell$. It is clearly a representation of $\mathrm{GL}(V)$. If $\mathcal{V}$ is a vector bundle of rank $n$, the subbundle $\mathrm{T}^{\ell}(\mathcal{V}) \subset \mathcal{V}^{\otimes \ell}$ is defined to be the associated bundle 
of the frame bundle of $\mathcal{V}$ (which is a principal GL(n)-bundle) through the representation $\mathrm{T}^{\ell}(V)$.

By sending any $e_{1}^{k_{1}} e_{2}^{k_{2}} \cdots e_{n}^{k_{n}} \in \operatorname{Sym}^{\ell}(V)$ to $v\left(k_{1}, \ldots, k_{n}\right)$, we have

$$
\operatorname{Sym}^{\ell}(V) \rightarrow \mathrm{T}^{\ell}(V)
$$

which is an isomorphism in characteristic zero. When $\operatorname{char}(k)=p>0$, we have $v\left(k_{1}, \ldots, k_{n}\right)=0$ if one of $k_{1}, \ldots, k_{n}$ is bigger than $p-1$. Thus (3.11) is not injective when $\ell \geq p$, and $\mathrm{T}^{\ell}(V)$ is isomorphic to the quotient of $\operatorname{Sym}^{\ell}(V)$ by the relations $e_{i}^{p}=0,1 \leq i \leq n$. In particular,

$$
\mathrm{T}^{\ell}(V) \cong \operatorname{Sym}^{\ell}(V) \text { when } 0<\ell<p
$$

and $\mathrm{T}^{\ell}(V)=0$ if $\ell>n(p-1)$. For any $0<\ell \leq n(p-1), \mathrm{T}^{\ell}(V)$ is a simple representation of highest weight

$$
(\overbrace{p-1, \cdots, p-1}^{a}, \overbrace{b, 0, \cdots, 0}^{n-a}), \quad \text { where } \ell=(p-1) a+b, 0 \leq b<p-1
$$

and is called a 'Truncated symmetric power' (cf. 3]). In next proposition, we will describe $\mathrm{T}^{\ell}(V)$ using symmetric powers and exterior powers. The case of GL(2) is extremely simple, it is a tensor product of symmetric powers and exterior powers. In general, let $F^{*} V$ denote the Frobenius twist of the standard representation $V$ of $\mathrm{GL}(n)$ through the homomorphism GL $(n) \rightarrow \mathrm{GL}(n)\left(\left(a_{i j}\right)_{n \times n} \rightarrow\left(a_{i j}^{p}\right)_{n \times n}\right)$, we have only a resolution of $\mathrm{T}^{\ell}(V)$ using symmetric powers of $V$ and exterior powers of $F^{*} V$. Fix a basis $e_{1}, \ldots, e_{n}$ of $V$, we define the $k$-linear maps

$$
\operatorname{Sym}^{\ell-q \cdot p}(V) \otimes_{k} \bigwedge^{q}(V) \stackrel{\phi}{\rightarrow} \operatorname{Sym}^{\ell-(q-1) \cdot p}(V) \otimes_{k} \bigwedge^{q-1}(V)
$$

such that for any $h=f_{\ell-q \cdot p} \otimes e_{k_{1}} \wedge \cdots \wedge e_{k_{q}}\left(k_{1}<\cdots<k_{q}\right)$, we have

$$
\phi(h)=\sum_{i=1}^{q}(-1)^{i-1} e_{k_{i}}^{p} f_{\ell-q \cdot p} \otimes e_{k_{1}} \wedge \cdots \wedge \hat{e}_{k_{i}} \wedge \cdots \wedge e_{k_{q}}
$$

Proposition 3.5. (i) When $n=2$, as $\mathrm{GL}(2)$-representations, we have

$$
\mathrm{T}^{\ell}(V)= \begin{cases}\operatorname{Sym}^{\ell}(V) & \text { when } \ell<p \\ \operatorname{Sym}^{2(p-1)-\ell}(V) \otimes \operatorname{det}(V)^{\ell-(p-1)} & \text { when } \ell \geq p\end{cases}
$$


(ii) Let $\ell(p) \geq 0$ be the unique integer such that $0 \leq \ell-\ell(p) \cdot p<p$. Then, in the category of $\mathrm{GL}(n)$-representations, we have exact sequence

$$
\begin{aligned}
& 0 \rightarrow \operatorname{Sym}^{\ell-\ell(p) \cdot p}(V) \otimes_{k} \bigwedge^{\ell(p)}\left(F^{*} V\right) \stackrel{\phi}{\rightarrow} \operatorname{Sym}^{\ell-(\ell(p)-1) \cdot p}(V) \otimes_{k} \bigwedge_{q-1}^{\ell(p)-1}\left(F^{*} V\right) \\
& \rightarrow \cdots \rightarrow \operatorname{Sym}^{\ell-q \cdot p}(V) \otimes_{k} \bigwedge^{q}\left(F^{*} V\right) \stackrel{\phi}{\rightarrow} \operatorname{Sym}^{\ell-(q-1) \cdot p}(V) \otimes_{k} \bigwedge^{q-1}\left(F^{*} V\right) \\
& \rightarrow \cdots \rightarrow \operatorname{Sym}^{\ell-p}(V) \otimes_{k} F^{*} V \stackrel{\phi}{\rightarrow} \operatorname{Sym}^{\ell}(V) \rightarrow \mathrm{T}^{\ell}(V) \rightarrow 0 .
\end{aligned}
$$

Proof. (i) When $\ell<p, \mathrm{~T}^{\ell}(V)=\operatorname{Sym}^{\ell}(V)$ follows the construction. When $\ell \geq p$, the simple representation $\mathrm{T}^{\ell}(V)$ has highest weight

$$
(p-1, \ell-p+1)=(2 p-2-\ell, 0)+(\ell-p+1) \cdot(1,1)
$$

where $(2 p-2-\ell, 0)$ and $(1,1)$ are the highest weights of the simple representations $\operatorname{Sym}^{2 p-2-\ell}(V)$ and $\wedge^{2}(V)=\operatorname{det}(V)$ respectively. Thus

$$
\mathrm{T}^{\ell}(V)=\operatorname{Sym}^{2(p-1)-\ell}(V) \otimes \operatorname{det}(V)^{\ell-(p-1)} .
$$

(ii) The elements $e_{1}^{p}, e_{2}^{p}, \ldots, e_{n}^{p} \in \operatorname{Sym}^{\bullet}(V)$ form clearly a regular sequence for $\operatorname{Sym}^{\bullet}(V)$, thus the Koszul complex $K_{\bullet}\left(e_{1}^{p}, \ldots, e_{n}^{p}\right)$ of $\operatorname{Sym}^{\bullet}(V)$-modules is a resolution of

$$
\frac{\operatorname{Sym}^{\bullet}(V)}{\left(e_{1}^{p}, e_{2}^{p}, \ldots, e_{n}^{p}\right) \operatorname{Sym}^{\bullet}(V)}
$$

where $K_{1}=\operatorname{Sym}^{\bullet}(V) \otimes_{k} V$ with basis $1 \otimes_{k} e_{1}, \ldots, 1 \otimes_{k} e_{n}$ and $K_{i}=$ $\wedge^{i} K_{1}$. Notice $\wedge^{i} K_{1} \cong \operatorname{Sym}^{\bullet}(V) \otimes_{k} \wedge^{i} V$ (as $\operatorname{Sym} \bullet(V)$-modules), the sequence in the proposition is exact in the category of $k$-linear spaces (This was pointed out by Manfred Lehn).

We only need to show the $k$-linear maps $\phi$ in (3.13) are maps of $\mathrm{GL}(n)$-representations if $\wedge^{\cdot} V$ is twisted by Frobenius. It is enough to show, for any $A=\left(a_{i j}\right)_{n \times n} \in \mathrm{GL}(n)$ and $h=1 \otimes e_{k_{1}} \wedge \cdots \wedge e_{k_{q}}$, that

$$
\phi(A \cdot h)=A \cdot \phi(h)
$$

To simplify notation, we assume $h=1 \otimes e_{1} \wedge \cdots \wedge e_{q}$, then

$$
\begin{aligned}
& A \cdot h=1 \otimes \sum_{k_{1}<\cdots<k_{q}} D\left(\begin{array}{c}
k_{1}, k_{2}, \ldots, k_{q} \\
1,2, \ldots, q
\end{array}\right) e_{k_{1}} \wedge \cdots \wedge e_{k_{q}}, \quad \text { where }
\end{aligned}
$$

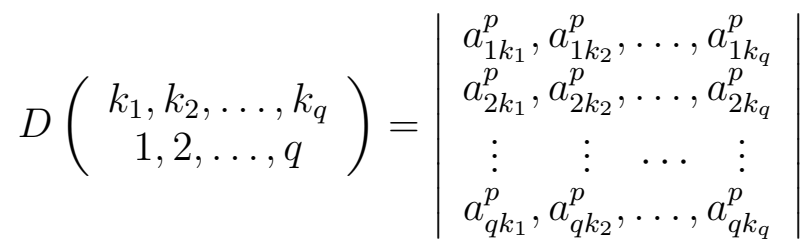


Then, by definition of $\phi$, we have

$$
\begin{aligned}
& \phi(A \cdot h)=\sum_{k_{1}<\cdots<k_{q}} \sum_{i=1}^{q}(-1)^{i-1} e_{k_{i}}^{p} \otimes_{k} D\left(\begin{array}{c}
k_{1}, k_{2}, \ldots, k_{q} \\
1,2, \ldots, q
\end{array}\right) e_{k_{1}} \wedge \cdots \wedge \hat{e}_{k_{i}} \wedge \cdots \wedge e_{k_{q}} \\
& =\sum_{k_{1}<\cdots<k_{q}} \sum_{i=1}^{q} e_{k_{i}}^{p} \otimes_{k} \sum_{j=1}^{q}(-1)^{j-1} a_{j k_{i}}^{p} D\left(\begin{array}{c}
k_{1}, \ldots, \hat{k}_{i}, \ldots, k_{q} \\
1, \ldots, \hat{j}, \ldots, q
\end{array}\right) e_{k_{1}} \wedge \cdots \wedge \hat{e}_{k_{i}} \wedge \cdots \wedge e_{k_{q}} \\
& =\sum_{j=1}^{q}(-1)^{j-1} \sum_{k_{1}<\cdots<k_{q}} \sum_{i=1}^{q} a_{j k_{i}}^{p} e_{k_{i}}^{p} \otimes_{k} D\left(\begin{array}{c}
k_{1}, \ldots, \hat{k}_{i}, \ldots, k_{q} \\
1, \ldots, \hat{j}, \ldots, q
\end{array}\right) e_{k_{1}} \wedge \cdots \wedge \hat{e}_{k_{i}} \wedge \cdots \wedge e_{k_{q}} .
\end{aligned}
$$

On the other hand, we will show

$$
\begin{gathered}
\sum_{k_{1}<\cdots<k_{q}} \sum_{i=1}^{q} a_{j k_{i}}^{p} e_{k_{i}}^{p} \otimes_{k} D\left(\begin{array}{c}
k_{1}, \ldots, \hat{k}_{i}, \ldots, k_{q} \\
1, \ldots, \hat{j}, \ldots, q
\end{array}\right) e_{k_{1}} \wedge \cdots \wedge \hat{e}_{k_{i}} \wedge \cdots \wedge e_{k_{q}} \\
=\left(\sum_{i=1}^{n} a_{j i}^{p} e_{i}^{p}\right) \otimes_{k}\left(\sum_{i=1}^{n} a_{1 i}^{p} e_{i}\right) \wedge \ldots\left(\begin{array}{c}
n \\
\sum_{i=1}^{n} a_{j i}^{p} e_{i}
\end{array}\right) \cdots \wedge\left(\sum_{i=1}^{n} a_{q i}^{p} e_{i}\right) \\
-\sum_{i_{1}<\cdots<i_{q-1}}\left(\sum_{k=1}^{q-1} a_{j i_{k}}^{p} e_{i_{k}}^{p}\right) \otimes_{k} D\left(\begin{array}{c}
i_{1}, \ldots, i_{q-1} \\
1, \ldots \hat{j} \ldots, q
\end{array}\right) e_{i_{1}} \wedge \cdots \wedge e_{i_{q-1}} \\
\text { and } \sum_{j=1}^{q}(-1)^{j-1} a_{j i_{k}}^{p} \cdot D\left(\begin{array}{c}
i_{1}, \ldots, i_{q-1} \\
1, \ldots \hat{j} \ldots, q
\end{array}\right)=0(1 \leq k \leq q-1) . \text { Thus } \\
\phi(A \cdot h)=A \cdot \phi(h) .
\end{gathered}
$$

In fact, the second equality corresponds to developing a determinant having the $i_{k}$-th column repeated. To show the first equality, write

$$
\begin{aligned}
& \left(\sum_{i=1}^{n} a_{j i}^{p} e_{i}^{p}\right) \otimes_{k}\left(\sum_{i=1}^{n} a_{1 i}^{p} e_{i}\right) \wedge \cdots \wedge\left(\widehat{\sum_{i=1}^{n} a_{j i}^{p}} e_{i}\right) \wedge \cdots \wedge\left(\sum_{i=1}^{n} a_{q i}^{p} e_{i}\right) \\
& =\sum_{i_{1}<\cdots<i_{q-1}}\left(\sum_{i=1}^{n} a_{j i}^{p} e_{i}^{p}\right) \otimes_{k} D\left(\begin{array}{c}
i_{1}, \ldots, i_{q-1} \\
1, \ldots \hat{j} \ldots, q
\end{array}\right) e_{i_{1}} \wedge \cdots \wedge e_{i_{q-1}}
\end{aligned}
$$


For given $i_{1}<\cdots<i_{q-1}$, let $S=\left\{i_{1}, \ldots, i_{q-1}\right\}$, write

$$
\begin{aligned}
& \left(\sum_{i=1}^{n} a_{j i}^{p} e_{i}^{p}\right) \otimes_{k} D\left(\begin{array}{c}
i_{1}, \ldots, i_{q-1} \\
1, \ldots \hat{j} \ldots, q
\end{array}\right) e_{i_{1}} \wedge \cdots \wedge e_{i_{q-1}}= \\
& \sum_{t \notin S} a_{j t}^{p} e_{t}^{p} \otimes_{k} D\left(\begin{array}{c}
i_{1}, \ldots, i_{q-1} \\
1, \ldots \hat{j} \ldots, q
\end{array}\right) e_{i_{1}} \wedge \cdots \wedge e_{i_{q-1}}+ \\
& \sum_{k=1}^{q-1} a_{j i_{k}}^{p} e_{i_{k}}^{p} \otimes_{k} D\left(\begin{array}{c}
i_{1}, \ldots, i_{q-1} \\
1, \ldots \hat{j} \ldots, q
\end{array}\right) e_{i_{1}} \wedge \cdots \wedge e_{i_{q-1}}
\end{aligned}
$$

notice that for any $t \notin S$ there is a unique $k_{1}<\cdots<k_{q}$ with $k_{i}=t$ such that $\left(k_{1}, \ldots, \hat{k}_{i}, \ldots, k_{q}\right)=\left(i_{1}, \ldots, i_{q-1}\right)$, we have

$$
\begin{aligned}
& \sum_{t \notin S} a_{j t}^{p} e_{t}^{p} \otimes_{k} D\left(\begin{array}{c}
i_{1}, \ldots, i_{q-1} \\
1, \ldots \hat{j} \ldots, q
\end{array}\right) e_{i_{1}} \wedge \cdots \wedge e_{i_{q-1}}= \\
& \sum_{k_{1}<\cdots<k_{q}} a_{j k_{i}}^{p} e_{k_{i}}^{p} \otimes_{k} D\left(\begin{array}{c}
k_{1}, \ldots, \hat{k}_{i}, \ldots, k_{q} \\
1, \ldots, \hat{j}, \ldots, q
\end{array}\right) e_{k_{1}} \wedge \cdots \wedge \hat{e}_{k_{i}} \wedge \cdots \wedge e_{k_{q}}
\end{aligned}
$$

where the summation is taken for all $k_{1}<\cdots<k_{q}$ satisfying

$$
\left(k_{1}, \ldots, \hat{k}_{i}, \ldots, k_{q}\right)=\left(i_{1}, \ldots, i_{q-1}\right) .
$$

Then, taking summation for all $i_{1}<\cdots<i_{q-1}$ and exchange the order of two summations, we got the claimed equality.

Lemma 3.6. With the notation in Definition 3.4, the composition

$$
\nabla^{\ell}: I_{\ell} / I_{\ell+1} \rightarrow\left(\Omega_{X}^{1}\right)^{\otimes \ell}
$$

of the $\mathcal{O}_{X}$-morphisms in Lemma 3.3 (ii) has image $\mathrm{T}^{\ell}\left(\Omega_{X}^{1}\right) \subset\left(\Omega_{X}^{1}\right)^{\otimes \ell}$.

Proof. It is enough to prove the lemma locally. By Lemma 3.2, $I_{\ell} / I_{\ell+1}$ is locally generated by

$$
\left\{\alpha_{1}^{k_{1}} \cdots \alpha_{n}^{k_{n}} \mid k_{1}+\cdots+k_{n}=\ell\right\} .
$$

By using formula (3.6) and the formula of permutations with repeated objects, we have

$$
\nabla^{\ell}\left(\alpha_{1}^{k_{1}} \cdots \alpha_{n}^{k_{n}}\right)=(-1)^{\ell} \sum_{\sigma \in \mathrm{S}_{\ell}}\left(\mathrm{d} x_{1}^{\otimes k_{1}} \otimes \cdots \mathrm{d} x_{n}^{\otimes k_{n}}\right) \cdot \sigma
$$

which implies that $\nabla^{\ell}\left(I_{\ell} / I_{\ell+1}\right)=\mathrm{T}^{\ell}\left(\Omega_{X}^{1}\right) \subset\left(\Omega_{X}^{1}\right)^{\otimes \ell}$.

Theorem 3.7. The filtration defined in Definition 3.1 is

$$
0=V_{n(p-1)+1} \subset V_{n(p-1)} \subset \cdots \subset V_{1} \subset V_{0}=V=F^{*}\left(F_{*} W\right)
$$

which has the following properties 
(i) $\nabla\left(V_{\ell+1}\right) \subset V_{\ell} \otimes \Omega_{X}^{1}$ for $\ell \geq 1$, and $V_{0} / V_{1} \cong W$.

(ii) $V_{\ell} / V_{\ell+1} \stackrel{\nabla}{\rightarrow}\left(V_{\ell-1} / V_{\ell}\right) \otimes \Omega_{X}^{1}$ are injective morphisms of vector bundles for $1 \leq \ell \leq n(p-1)$, which induced isomorphisms

$$
\nabla^{\ell}: V_{\ell} / V_{\ell+1} \cong W \otimes_{\mathcal{O}_{X}} \mathrm{~T}^{\ell}\left(\Omega_{X}^{1}\right), \quad 0 \leq \ell \leq n(p-1) .
$$

The vector bundle $\mathrm{T}^{\ell}\left(\Omega_{X}^{1}\right)$ is suited in the exact sequence

$$
\begin{aligned}
0 & \rightarrow \operatorname{Sym}^{\ell-\ell(p) \cdot p}\left(\Omega_{X}^{1}\right) \otimes F^{*} \Omega_{X}^{\ell(p)} \stackrel{\phi}{\rightarrow} \operatorname{Sym}^{\ell-(\ell(p)-1) \cdot p}\left(\Omega_{X}^{1}\right) \otimes F^{*} \Omega_{X}^{\ell(p)-1} \\
& \rightarrow \cdots \rightarrow \operatorname{Sym}^{\ell-q \cdot p}\left(\Omega_{X}^{1}\right) \otimes F^{*} \Omega_{X}^{q} \stackrel{\phi}{\rightarrow} \operatorname{Sym}^{\ell-(q-1) \cdot p}\left(\Omega_{X}^{1}\right) \otimes F^{*} \Omega_{X}^{q-1} \\
& \rightarrow \cdots \rightarrow \operatorname{Sym}^{\ell-p}\left(\Omega_{X}^{1}\right) \otimes F^{*} \Omega_{X}^{1} \stackrel{\phi}{\rightarrow} \operatorname{Sym}^{\ell}\left(\Omega_{X}^{1}\right) \rightarrow \mathrm{T}^{\ell}\left(\Omega_{X}^{1}\right) \rightarrow 0
\end{aligned}
$$

where $\ell(p) \geq 0$ is the integer such that $\ell-\ell(p) \cdot p<p$.

Proof. It is a local problem to prove the theorem. Thus $V_{n(p-1)+1}=0$ follows from Lemma 3.2. (i) is nothing but the definition. (ii) follows from Lemma 3.3, Proposition 3.5 and Lemma 3.6.

Corollary 3.8. When $\operatorname{dim}(X)=2$, we have

$$
V_{\ell} / V_{\ell+1}= \begin{cases}W \otimes \operatorname{Sym}^{\ell}\left(\Omega_{X}^{1}\right) & \text { when } \ell<p \\ W \otimes \operatorname{Sym}^{2(p-1)-\ell}\left(\Omega_{X}^{1}\right) \otimes \omega_{X}^{\ell-(p-1)} & \text { when } \ell \geq p\end{cases}
$$

Proof. It follows from (i) of Proposition 3.5 .

\section{STABILITY IN HIGHER DIMENSIONAL CASE}

Let $X$ be a smooth projective variety over $k$ of dimension $n$ and $\mathrm{H}$ a fixed ample divisor on $X$. For a torsion free sheaf $\mathcal{E}$ on $X$, we define

$$
\mu(\mathcal{E})=\frac{c_{1}(\mathcal{E}) \cdot \mathrm{H}^{n-1}}{\operatorname{rk}(\mathcal{E})}
$$

Definition 4.1. A torsion free sheaf $\mathcal{E}$ on $X$ is called semistable (resp. stable) if, for any $0 \neq \mathcal{E}^{\prime} \subset \mathcal{E}$, we have

$$
\mu\left(\mathcal{E}^{\prime}\right) \leq \mu(\mathcal{E}) \quad\left(\text { resp. } \mu\left(\mathcal{E}^{\prime}\right)<\mu(\mathcal{E})\right) .
$$

For any torsion free sheaf $E$ on $X$, there is a unique filtration, the so-called Harder-Narasimhan filtration

$$
0=E_{0} \subset E_{1} \subset \cdots \subset E_{k}=E
$$

such that $E_{i} / E_{i-1}(1 \leq i \leq k)$ are semistable torsion free sheaves and

$$
\mu_{\max }(E):=\mu\left(E_{1}\right)>\mu\left(E_{2} / E_{1}\right)>\cdots>\mu\left(E_{k} / E_{k-1}\right):=\mu_{\min }(E) .
$$

The instability of $E$ was defined as

$$
\mathrm{I}(E)=\mu_{\max }(E)-\mu_{\min }(E) .
$$


Then it is easy to see that for any subsheaf $F \subset E$ we have

$$
\mu(F)-\mu(E) \leq \mathrm{I}(E)=\mu_{\max }(E)-\mu_{\min }(E) .
$$

Let $F: X \rightarrow X_{1}$ be the relative $k$-linear Frobenius morphism and $W$ a vector bundle of rank $r$ on $X$.

Lemma 4.2. Let $c_{1}\left(\Omega_{X}^{1}\right)=K_{X}$. Then, in the chow group $\operatorname{Ch}\left(X_{1}\right)_{\mathbb{Q}}$,

$$
\begin{gathered}
c_{1}\left(F_{*} W\right)=\frac{r\left(p^{n}-p^{n-1}\right)}{2} K_{X_{1}}+p^{n-1} c_{1}(W), \\
\mu\left(F^{*} F_{*} W\right)=p \cdot \mu\left(F_{*} W\right)=\frac{p-1}{2} K_{X} \cdot \mathrm{H}^{n-1}+\mu(W) .
\end{gathered}
$$

Proof. The proof is just an application of Riemann-Roch theorem. Indeed, by Grothendieck-Riemann-Roch theorem, we have

$$
c_{1}\left(F_{*} W\right)=\frac{r p^{n}}{2} K_{X}+F_{*}\left(c_{1}(W)-\frac{r}{2} K_{X}\right) .
$$

We remark here that for any irreducible subvariety $Y \subset X$, its image $F_{X}(Y) \subset X$ (under the absolute Frobenius $F_{X}: X \rightarrow X$ ) equals to $Y$, and the induced morphism $F_{X}: Y \rightarrow F_{X}(Y)=Y$ is nothing but the absolute Frobenius morphism $F_{Y}: Y \rightarrow Y$ (which has degree $p^{\operatorname{dim}(Y)}$ ). In particular, $F_{*}\left(c_{1}(W)-\frac{r}{2} K_{X}\right)=p^{n-1}\left(c_{1}(W)-\frac{r}{2} K_{X_{1}}\right)$ proves (4.2). That $\mu\left(F^{*} F_{*} W\right)=p \cdot \mu\left(F_{*} W\right)$ also follows from this remark.

Let $V=F^{*} F_{*} W$, recall Theorem 3.7 , we have the canonical filtration

$$
0=V_{n(p-1)+1} \subset V_{n(p-1)} \subset \cdots \subset V_{1} \subset V_{0}=V=F^{*}\left(F_{*} W\right)
$$

with $V_{\ell} / V_{\ell+1} \cong W \otimes_{\mathcal{O}_{X}} \mathrm{~T}^{\ell}\left(\Omega_{X}^{1}\right)$.

Lemma 4.3. With the same notation in Theorem 3.7, we have

$$
\begin{gathered}
c_{1}\left(\mathrm{~T}^{\ell}\left(\Omega_{X}^{1}\right)\right)=\frac{\ell}{n}\left(\sum_{q=0}^{\ell(p)}(-1)^{q} C_{n}^{q} \cdot C_{n+\ell-q-1}^{\ell-q p}\right) K_{X} \\
\operatorname{rk}\left(\mathrm{T}^{\ell}\left(\Omega_{X}^{1}\right)\right)=\sum_{q=0}^{\ell(p)}(-1)^{q} C_{n}^{q} \cdot C_{n+\ell-q-1}^{\ell-q p} .
\end{gathered}
$$

In particular, we have $\mu\left(\mathrm{T}^{\ell}\left(\Omega_{X}^{1}\right)\right)=\frac{\ell}{n} K_{X} \cdot \mathrm{H}^{n-1}$.

Proof. The formula of $\operatorname{rk}\left(\mathrm{T}^{\ell}\left(\Omega_{X}^{1}\right)\right)$ follows directly from the exact sequence in Theorem 3.7 (ii). To compute $c_{1}\left(\mathrm{~T}^{\ell}\left(\Omega_{X}^{1}\right)\right)$, we use the fact that for any vector bundle $E$ of rank $n$, we have

$$
\begin{gathered}
c_{1}\left(\operatorname{Sym}^{q}(E)\right)=C_{n+q-1}^{q-1} \cdot c_{1}(E) \\
c_{1}\left(\wedge^{q} E\right)=C_{n-1}^{q-1} \cdot c_{1}(E) .
\end{gathered}
$$


Then, use the exact sequence in Theorem 3.7 (ii) and note that

$$
c_{1}\left(F^{*} \Omega_{X}^{q}\right)=p \cdot c_{1}\left(\Omega_{X}^{q}\right),
$$

we have the formula (4.5) of $c_{1}\left(\mathrm{~T}^{\ell}\left(\Omega_{X}^{1}\right)\right)$.

Let $\mathcal{E} \subset F_{*} W$ be a nontrivial subsheaf, the canonical filtration (4.4) induces the filtration (we assume $V_{m} \cap F^{*} \mathcal{E} \neq 0$ )

$$
0 \subset V_{m} \cap F^{*} \mathcal{E} \subset \cdots \subset V_{1} \cap F^{*} \mathcal{E} \subset V_{0} \cap F^{*} \mathcal{E}=F^{*} \mathcal{E} .
$$

Lemma 4.4. In the induced filtration (4.8), let

$$
\mathcal{F}_{\ell}:=\frac{V_{\ell} \cap F^{*} \mathcal{E}}{V_{\ell+1} \cap F^{*} \mathcal{E}} \subset \frac{V_{\ell}}{V_{\ell+1}}, \quad r_{\ell}=\operatorname{rk}\left(\mathcal{F}_{\ell}\right) .
$$

Then there is an injective morphism $\mathcal{F}_{\ell} \stackrel{\nabla}{\rightarrow} \mathcal{F}_{\ell-1} \otimes \Omega_{X}^{1}$ and

$$
\begin{aligned}
\mu\left(F_{*} W\right)-\mu(\mathcal{E}) \geq & \frac{K_{X} \cdot \mathrm{H}^{n-1}}{n p \cdot \operatorname{rk}(\mathcal{E})} \sum_{\ell=0}^{m}\left(\frac{p-1}{2} n-\ell\right) \cdot r_{\ell} \\
& -\frac{1}{p} \sum_{\ell=0}^{m} \frac{r_{\ell} \cdot \mathrm{I}\left(W \otimes \mathrm{T}^{\ell}\left(\Omega_{X}^{1}\right)\right)}{\operatorname{rk}(\mathcal{E})}
\end{aligned}
$$

the equality holds if and only if equalities hold in the inequalities

$$
\mu\left(\mathcal{F}_{\ell}\right)-\mu\left(V_{\ell} / V_{\ell+1}\right) \leq \mathrm{I}\left(W \otimes \mathrm{T}^{\ell}\left(\Omega_{X}^{1}\right)\right) \quad(0 \leq \ell \leq m) .
$$

Proof. The injective morphisms $V_{\ell} / V_{\ell+1} \stackrel{\nabla}{\rightarrow}\left(V_{\ell-1} / V_{\ell}\right) \otimes \Omega_{X}^{1}$ in Theorem 3.7 (ii) induces clearly the injective morphisms

$$
\mathcal{F}_{\ell} \stackrel{\nabla}{\rightarrow} \mathcal{F}_{\ell-1} \otimes \Omega_{X}^{1}, \quad \ell=1, \ldots, m .
$$

To show (4.9) $)$, note $\mu\left(F_{*} W\right)-\mu(\mathcal{E})=\frac{1}{p}\left(\mu\left(F^{*} F_{*} W\right)-\mu\left(F^{*} \mathcal{E}\right)\right)$ and

$$
\mu\left(F^{*} \mathcal{E}\right)=\frac{1}{\operatorname{rk}(\mathcal{E})} \sum_{\ell=0}^{m} r_{\ell} \cdot \mu\left(\mathcal{F}_{\ell}\right)
$$

using Lemma 4.2, we have

$$
\begin{gathered}
\mu\left(F^{*} F_{*} W\right)-\mu\left(F^{*} \mathcal{E}\right)= \\
\frac{1}{\operatorname{rk}(\mathcal{E})} \sum_{\ell=0}^{m} r_{\ell}\left(\frac{p-1}{2} K_{X} \cdot \mathrm{H}^{n-1}+\mu(W)-\mu\left(\mathcal{F}_{\ell}\right)\right) .
\end{gathered}
$$

For $\mathcal{F}_{\ell} \subset V_{\ell} / V_{\ell+1}=W \otimes \mathrm{T}^{\ell}\left(\Omega_{X}^{1}\right)(0 \leq \ell \leq m)$, using Lemma 4.3,

$$
\mu\left(\mathcal{F}_{\ell}\right) \leq \mu(W)+\frac{\ell}{n} K_{X} \cdot \mathrm{H}^{n-1}+\mathrm{I}\left(W \otimes \mathrm{T}^{\ell}\left(\Omega_{X}^{1}\right)\right) .
$$

Substitute (4.12) into (4.11), one get (4.9) and the equality holds if and only if all of inequalities (4.12) become equalities. 
Let $K$ be a field of characteristic $p>0$, consider the $K$-algebra

$$
R=\frac{K\left[y_{1}, \cdots, y_{n}\right]}{\left(y_{1}^{p}, \cdots, y_{n}^{p}\right)}=\bigoplus_{\ell=0}^{n(p-1)} R^{\ell}
$$

where $R^{\ell}$ is the $K$-linear space generated by

$$
\left\{y_{1}^{k_{1}} \cdots y_{n}^{k_{n}} \mid k_{1}+\cdots+k_{n}=\ell, \quad 0 \leq k_{i} \leq p-1\right\} .
$$

The polynomial ring $\mathrm{P}=K\left[\partial_{y_{1}}, \cdots, \partial_{y_{n}}\right]$ acts on $R$ through partial derivations, which induces a P-module structure on $R$. Note that $\partial_{y_{i}}^{p}$ $(i=1,2, \ldots, n)$ act on $R$ trivially, the P-module structure is in fact a D-module, where

$$
\mathrm{D}=\frac{K\left[\partial_{y_{1}}, \cdots, \partial_{y_{n}}\right]}{\left(\partial_{y_{1}}^{p}, \cdots, \partial_{y_{n}}^{p}\right)}=K\left[t_{1}, t_{2}, \cdots, t_{n}\right]=\bigoplus_{\ell=0}^{n(p-1)} \mathrm{D}_{\ell}
$$

where $\mathrm{D}_{\ell}$ is the linear space of degree $\ell$ homogeneous elements and $t_{1}, t_{2}, \ldots, t_{n}$ are the classes of $\partial_{y_{1}}, \partial_{y_{2}}, \ldots, \partial_{y_{n}}$.

Lemma 4.5. Let $V \subset \mathrm{D}_{\ell}$ be a linear subspace. Then, when $\ell \leq \frac{n(p-1)}{2}$, there is a basis $\left\{d_{i} \in V\right\}$ of $V$ and monomials $\left\{\delta_{i} \in \mathrm{D}_{n(p-1)-2 \ell}\right\}$ such that $\left\{\delta_{i} d_{i} \in \mathrm{D}_{n(p-1)-\ell}\right\}$ are linearly independent.

Proof. We reduce firstly the lemma to the case when $V$ has a basis of monomials. Define the Lexicographic order on the set of monomials of $\mathrm{D}_{\ell}, \mathrm{D}_{n(p-1)-\ell}$ respectively. For any $v \in \mathrm{D}_{\ell}$, one can write uniquely

$$
v=\lambda_{v} m_{v}+\sum_{m>m_{v}} \lambda_{m} m
$$

where $0 \neq \lambda_{v}, \lambda_{m} \in K, m_{v}$ and $m$ are monomials of $\mathrm{D}_{\ell}$.

Let $\operatorname{dim}(V)=s$, then it is easy to see that there is a basis

$$
d_{i}=\lambda_{i} m_{i}+\sum_{m>m_{i}} \lambda_{i, m} m, \quad \lambda_{i} \neq 0, \quad(1 \leq i \leq s)
$$

of $V$ such that $\left\{m_{1}, \ldots, m_{s}\right\}$ are different monomials of $\mathrm{D}_{\ell}$. If there are monomials $\left\{\delta_{i} \in \mathrm{D}_{n(p-1)-2 \ell}\right\}_{1 \leq i \leq s}$ such that $\left\{\delta_{i} m_{i} \in \mathrm{D}_{n(p-1)-\ell}\right\}_{1 \leq i \leq s}$ are different monomials, then we claim that

$$
\left\{\delta_{i} d_{i} \in \mathrm{D}_{n(p-1)-\ell}\right\}_{1 \leq i \leq s}
$$

are linearly independent. To prove the claim, we only remark that for any monomials $m, m^{\prime} \in \mathrm{D}_{\ell}$ and monomial $\delta \in \mathrm{D}_{n(p-1)-2 \ell}$, we have

$$
m<m^{\prime} \Rightarrow \delta m<\delta m^{\prime} \quad \text { whenever } \delta m, \delta m^{\prime} \text { are nonzero. }
$$


Thus we have

$$
\delta_{i} d_{i}=\lambda_{i} \delta_{i} m_{i}+\sum_{\delta_{i} m>\delta m_{i}} \lambda_{i, m} \delta_{i} m \quad(1 \leq i \leq s),
$$

which are linearly independent.

If we identify the set of monomials of $\mathrm{D}_{\ell}$ with the set

$$
M^{\ell}=\left\{v=\left(v_{1}, \ldots, v_{n}\right) \mid 0 \leq v_{i} \leq p-1(1 \leq i \leq n), \quad \sum_{i=1}^{n} v_{i}=\ell\right\} .
$$

Then the lemma is equivalent to the existence of an injective map

$$
\varphi: M^{\ell} \rightarrow M^{n(p-1)-\ell}
$$

such that for any $v \in M^{\ell}$, we have $v \leq \varphi(v): v_{i} \leq \varphi(v)_{i} \quad(1 \leq i \leq n)$. The existence of $\varphi$ is a special case of the following lemma.

For any $\left(a_{1}, \ldots, a_{n}\right) \in \mathbb{Z}_{\geq 0}^{n}$, let $M_{n}^{\ell}\left(a_{1}, \ldots, a_{n}\right)$ be the set

$$
\left\{v=\left(v_{1}, \ldots, v_{n}\right) \mid 0 \leq v_{i} \leq a_{i}(1 \leq i \leq n), \sum_{i=1}^{n} v_{i}=\ell\right\}
$$

For any $v \in M_{n}^{\ell}\left(a_{1}, \ldots, a_{n}\right)$ and $v^{\prime} \in M_{n}^{\ell^{\prime}}\left(a_{1}, \ldots, a_{n}\right)$, by $v \leq v^{\prime}$, we mean $v_{i} \leq v_{i}^{\prime}(1 \leq i \leq n)$. Then we have the following lemma, its proof was suggested by Fusheng Leng

Lemma 4.6. Let $\sigma=\sum_{i=1}^{n} a_{i}$. Then, when $\ell \leq \frac{1}{2} \sigma$, there exists an injective map $\varphi: M_{n}^{\ell}\left(a_{1}, \ldots, a_{n}\right) \rightarrow M_{n}^{\sigma-\ell}\left(a_{1}, \ldots, a_{n}\right)$ such that

$$
v \leq \varphi(v), \quad \forall v \in M_{n}^{\ell}\left(a_{1}, \ldots, a_{n}\right) .
$$

Proof. The strategy of proof is to do induction for $n$ and $\sigma$. The lemma is clearly true when $n=1$. Assume the lemma is true for $n-1$. To show the lemma for $n$, we do induction for $\sigma$. The lemma is trivially true for any $n$ when $\sigma=1$. Thus we can assume $n \geq 2$ and $\sigma \geq 2$.

Without loss of generality, we assume $a_{n-1}>0$ and $a_{n}>0$. Let

$$
\begin{aligned}
S^{\ell} & =\left\{v \in M_{n}^{\ell}\left(a_{1}, \ldots, a_{n}\right) \mid v_{n-1}=a_{n-1} \text { or } v_{n}=0\right\}, \\
S^{\sigma-\ell} & =\left\{v \in M_{n}^{\sigma-\ell}\left(a_{1}, \ldots, a_{n}\right) \mid v_{n-1}=a_{n-1} \text { or } v_{n}=0\right\},
\end{aligned}
$$

$C^{\ell}=M_{n}^{\ell}\left(a_{1}, \ldots, a_{n}\right) \backslash S^{\ell}$ and $C^{\sigma-\ell}=M_{n}^{\sigma-\ell}\left(a_{1}, \ldots, a_{n}\right) \backslash S^{\sigma-\ell}$. We will show the existence of injective maps

$$
\varphi_{1}: S^{\ell} \rightarrow S^{\sigma-\ell}, \quad \varphi_{2}: C^{\ell} \rightarrow C^{\sigma-\ell}
$$


with $v \leq \varphi_{1}(v), v \leq \varphi_{2}(v)\left(\forall v \in S^{\ell}, \forall v \in C^{\ell}\right)$ by induction of $n, \sigma$ respectively. In order to use the induction, we identify $S^{\ell}$ (resp. $S^{\sigma-\ell}$ ) with $M_{n-1}^{\ell}\left(a_{1}, \ldots, a_{n-1}+a_{n}\right)\left(\right.$ resp. $\left.M_{n-1}^{\sigma-\ell}\left(a_{1}, \ldots, a_{n-1}+a_{n}\right)\right)$ by

$$
f_{\ell}: S^{\ell} \rightarrow M_{n-1}^{\ell}\left(a_{1}, \ldots, a_{n-1}+a_{n}\right), \quad f_{\ell}(v)=\left(v_{1}, \ldots, v_{n-2}, v_{n-1}+v_{n}\right)
$$

(resp. $f_{\sigma-\ell}: S^{\sigma-\ell} \rightarrow M_{n-1}^{\sigma-\ell}\left(a_{1}, \ldots, a_{n-1}+a_{n}\right)$ ). Indeed, $f_{\ell}$ (resp. $f_{\sigma-\ell}$ ) is a bijective map. To see the injectivity of $f_{\ell}$, if $f_{\ell}(v)=f_{\ell}\left(v^{\prime}\right)$, then $v_{i}=v_{i}^{\prime}(1 \leq i \leq n-2)$ and $v_{n-1}+v_{n}=v_{n-1}^{\prime}+v_{n}^{\prime}$. We claim that $v_{n-1}+v_{n}=v_{n-1}^{\prime}+v_{n}^{\prime}$ implies $v_{n}=v_{n}^{\prime}$ (thus $v_{n-1}=v_{n-1}^{\prime}$ ) since $v, v^{\prime} \in S^{\ell}$. Indeed, if $v_{n}=0$ then $v_{n}^{\prime}=0$, otherwise $v_{n-1}^{\prime}=a_{n-1}$ (by definition of $\left.S^{\ell}\right)$ and $v_{n-1}=a_{n-1}+v_{n}^{\prime}>a_{n-1}$ (a contradiction to the definition of $\left.M_{n}^{\ell}\left(a_{1}, \ldots, a_{n}\right)\right)$. Similarly, $v_{n}^{\prime}=0$ implies $v_{n}=0$. If both $v_{n}$ and $v_{n}^{\prime}$ are not zero, by definition of $S^{\ell}, v_{n-1}=a_{n-1}=v_{n-1}^{\prime}$, thus $v_{n}=v_{n}^{\prime}$. To see it being surjective, for any $w \in M_{n-1}^{\ell}\left(a_{1}, \ldots, a_{n-1}+a_{n}\right)$, notice that $w_{i} \leq a_{i}(1 \leq i \leq n-2)$ and $w_{n-1} \leq a_{n-1}+a_{n}$, we define

$$
v= \begin{cases}\left(w_{1}, \ldots, w_{n-2}, w_{n-1}, 0\right) & \text { if } w_{n-1} \leq a_{n-1} \\ \left(w_{1}, \ldots, w_{n-2}, a_{n-1}, w_{n-1}-a_{n-1}\right) & \text { if } w_{n-1}>a_{n-1}\end{cases}
$$

then $v \in S^{\ell}$ such that $f_{\ell}(v)=w$. Similarly, $f_{\sigma-\ell}$ is bijective.

By the inductive assumption for $n$, there exists an injective map

$$
\psi_{1}: M_{n-1}^{\ell}\left(a_{1}, \ldots, a_{n-1}+a_{n}\right) \rightarrow M_{n-1}^{\sigma-\ell}\left(a_{1}, \ldots, a_{n-1}+a_{n}\right)
$$

such that $v \leq \psi_{1}(v)\left(\forall v \in M_{n-1}^{\ell}\left(a_{1}, \ldots, a_{n-1}+a_{n}\right)\right)$. Then, we define

$$
\varphi_{1}=f_{\sigma-\ell}^{-1} \cdot \psi_{1} \cdot f_{\ell}: S^{\ell} \rightarrow S^{\sigma-\ell} .
$$

For any $v=\left(v_{1}, \ldots, v_{n}\right) \in S^{\ell}$, we need to show $v \leq \varphi_{1}(v)$. Let

$$
\psi_{1}\left(f_{\ell}(v)\right)=\left(w_{1}, \ldots, w_{n-2}, w_{n-1}\right) \in M_{n-1}^{\sigma-\ell}\left(a_{1}, \ldots, a_{n-1}+a_{n}\right) .
$$

Then $v_{i} \leq w_{i}(1 \leq i \leq n-2), v_{n-1}+v_{n} \leq w_{n-1}$ and

$$
\varphi_{1}(v)= \begin{cases}\left(w_{1}, \ldots, w_{n-2}, w_{n-1}, 0\right) & \text { if } w_{n-1} \leq a_{n-1} \\ \left(w_{1}, \ldots, w_{n-2}, a_{n-1}, w_{n-1}-a_{n-1}\right) & \text { if } w_{n-1}>a_{n-1}\end{cases}
$$

by the definition of $f_{\ell}, \psi_{1}$ and $f_{\sigma-\ell}$. Thus $v_{i} \leq \varphi_{1}(v)_{i}(1 \leq i \leq n-2)$. We still need to check $v_{n-1} \leq \varphi_{1}(v)_{n-1}$ and $v_{n} \leq \varphi_{1}(v)_{n}$. If $v_{n}=0$ (thus $\left.v_{n} \leq \varphi_{1}(v)_{n}\right)$, then $v_{n-1} \leq w_{n-1}$ (since $v_{n-1}+v_{n} \leq w_{n-1}$ ), thus

$$
v_{n-1} \leq \min \left\{w_{n-1}, a_{n-1}\right\} \leq \varphi_{1}(v)_{n-1} .
$$

If $v_{n} \neq 0$, by the definition of $S^{\ell}, v_{n-1}=a_{n-1}$, which implies

$$
a_{n-1}<a_{n-1}+v_{n}=v_{n-1}+v_{n} \leq w_{n-1} .
$$

Thus $\varphi_{1}(v)_{n-1}=a_{n-1}$ and $\varphi_{1}(v)_{n}=w_{n-1}-a_{n-1}=w_{n-1}-v_{n-1} \geq v_{n}$. 
Next we construct the injective map $\varphi_{2}: C^{\ell} \rightarrow C^{\sigma-\ell}$ by using induction for $\sigma$. By the definition of $C^{\ell}$ and $C^{\sigma-\ell}$, we have

$$
\begin{gathered}
C^{\ell}=\left\{v \in M_{n}^{\ell}\left(a_{1}, \ldots, a_{n}\right) \mid v_{n-1} \leq a_{n-1}-1, v_{n} \geq 1\right\} \\
C^{\sigma-\ell}=\left\{v^{\prime} \in M_{n}^{\sigma-\ell}\left(a_{1}, \ldots, a_{n}\right) \mid v_{n-1}^{\prime} \leq a_{n-1}-1, v_{n}^{\prime} \geq 1\right\} .
\end{gathered}
$$

Let $\bar{\sigma}=a_{1}+\cdots+a_{n-2}+\left(a_{n-1}-1\right)+\left(a_{n}-1\right)=\sigma-2$ and $\bar{\ell}=\ell-1$, we have the following clear identifications

$$
\begin{gathered}
\pi_{\ell}: C^{\ell} \rightarrow M_{n}^{\bar{\ell}}\left(a_{1}, \ldots, a_{n-2}, a_{n-1}-1, a_{n}-1\right) \\
\pi_{\sigma-\ell}: C^{\sigma-\ell} \rightarrow M_{n}^{\bar{\sigma}-\bar{\ell}}\left(a_{1}, \ldots, a_{n-2}, a_{n-1}-1, a_{n}-1\right)
\end{gathered}
$$

where $\pi_{\ell}(v)=\left(v_{1}, \ldots, v_{n-1}, v_{n}-1\right), \pi_{\sigma-\ell}\left(v^{\prime}\right)=\left(v_{1}^{\prime}, \ldots, v_{n-1}^{\prime}, v_{n}^{\prime}-1\right)$. Notice that $\bar{\ell} \leq \frac{1}{2} \bar{\sigma}$, by induction for $\sigma$, there exists an injective map

$\psi_{2}: M_{n}^{\bar{\ell}}\left(a_{1}, \ldots, a_{n-2}, a_{n-1}-1, a_{n}-1\right) \rightarrow M_{n}^{\bar{\sigma}-\bar{\ell}}\left(a_{1}, \ldots, a_{n-2}, a_{n-1}-1, a_{n}-1\right)$

such that $v \leq \psi_{2}(v)$ for any $v \in M_{n}^{\bar{\ell}}\left(a_{1}, \ldots, a_{n-2}, a_{n-1}-1, a_{n}-1\right)$. Let

$$
\varphi_{2}=\pi_{\sigma-\ell}^{-1} \cdot \psi_{2} \cdot \pi_{\ell}: C^{\ell} \rightarrow C^{\sigma-\ell} .
$$

For any $v=\left(v_{1}, \ldots, v_{n}\right) \in C^{\ell}$, we have to check that $v \leq \varphi_{2}(v)$. Let

$$
\psi_{2}\left(\pi_{\ell}(v)\right)=\left(w_{1}, \ldots, w_{n}\right) \in M_{n}^{\bar{\sigma}-\bar{\ell}}\left(a_{1}, \ldots, a_{n-2}, a_{n-1}-1, a_{n}-1\right),
$$

then $v_{i} \leq w_{i}(1 \leq i \leq n-1), v_{n}-1 \leq w_{n}$ and

$$
\varphi_{2}(v)=\left(w_{1}, \ldots, w_{n-1}, w_{n}+1\right) \in C^{\sigma-\ell}
$$

by the definition of $\pi_{\ell}, \psi_{2}$ and $\pi_{\sigma-\ell}$. Thus $v_{n} \leq w_{n}+1=\varphi_{2}(v)_{n}$ and we have shown the lemma.

Proposition 4.7. Let $V \subset R^{\ell}$ be a linear subspace, $\mathbb{L}\left(\mathrm{D}_{2 \ell-n(p-1)} \cdot V\right)$ be the linear subspace generated by $\mathrm{D}_{2 \ell-n(p-1)} \cdot V \subset R^{n(p-1)-\ell}$. Then,

$$
\operatorname{dim}(V) \leq \operatorname{dim} \mathbb{L}\left(\mathrm{D}_{2 \ell-n(p-1)} \cdot V\right) \quad \text { when } \frac{n(p-1)}{2} \leq \ell \leq n(p-1) .
$$

Proof. Let $\omega=y_{1}^{p-1} y_{2}^{p-1} \cdots y_{n}^{p-1} \in R^{n(p-1)}$. Then the D-module structure on $R$ induces surjective morphisms

$$
\phi_{\ell}: \mathrm{D}_{\ell} \stackrel{\cdot \omega}{\rightarrow} R^{n(p-1)-\ell}
$$

of linear spaces for any $0 \leq \ell \leq n(p-1)$. They must be isomorphisms since $\operatorname{dim}\left(\mathrm{D}_{\ell}\right)=\operatorname{dim}\left(R^{n(p-1)-\ell}\right)$. To show the equality of dimensions, it is enough to show

$$
\operatorname{dim}\left(\mathrm{D}_{\ell}\right) \geq \operatorname{dim}\left(R^{n(p-1)-\ell}\right)=\operatorname{dim}\left(\mathrm{D}_{n(p-1)-\ell}\right) \geq \operatorname{dim}\left(R^{\ell}\right)=\operatorname{dim}\left(\mathrm{D}_{\ell}\right) .
$$


The two inequalities hold because we have the surjective homomorphisms $\phi_{\ell}$ and $\phi_{n(p-1)-\ell}$. The two equalities hold because

$$
\bigoplus_{\ell=0}^{n(p-1)} R^{\ell}=R \cong \mathrm{D}=\bigoplus_{\ell=0}^{n(p-1)} \mathrm{D}_{\ell}
$$

as (graded) $K$-algebras. In particular,

$$
\phi_{n(p-1)-\ell}: \mathrm{D}_{n(p-1)-\ell} \rightarrow R^{\ell}, \quad \phi_{\ell}: \mathrm{D}_{\ell} \rightarrow R^{n(p-1)-\ell}
$$

are isomorphisms. Since $0 \leq \bar{\ell}=n(p-1)-\ell \leq \frac{n(p-1)}{2}$, we can use Lemma 4.5 for $V^{\prime}=\phi_{n(p-1)-\ell}^{-1}(V) \subset \mathrm{D}_{\bar{\ell}}=\mathrm{D}_{n(p-1)-\ell}$, thus there is a basis $\left\{d_{i} \in V^{\prime}\right\}_{1 \leq i \leq s}$ and monomials $\left\{\delta_{i} \in \mathrm{D}_{n(p-1)-2 \bar{\ell}}=\mathrm{D}_{2 \ell-n(p-1)}\right\}_{1 \leq i \leq s}$ such that $\left\{\delta_{i} d_{i} \in \mathrm{D}_{n(p-1)-\bar{\ell}}=\mathrm{D}_{\ell}\right\}_{1 \leq i \leq s}$ are linearly independent. Thus

$$
\left\{\phi_{\ell}\left(\delta_{i} d_{i}\right)=\delta_{i}\left(d_{i} \omega\right) \in \mathrm{D}_{2 \ell-n(p-1)} \cdot V \subset R^{n(p-1)-\ell}\right\}_{1 \leq i \leq s}
$$

are linearly independent, where $s=\operatorname{dim}\left(V^{\prime}\right)=\operatorname{dim}(V)$. We have proven the proposition.

Let $X$ be an irreducible smooth projective variety of dimension $n$ over an algebraically closed field $k$ with $\operatorname{char}(k)=p>0$. For any vector bundle $W$ on $X$, let

$$
\mathrm{I}(W, X)=\max \left\{\mathrm{I}\left(W \otimes \mathrm{T}^{\ell}\left(\Omega_{X}^{1}\right)\right) \mid 0 \leq \ell \leq n(p-1)\right\}
$$

be the maximal value of instabilities $\mathrm{I}\left(W \otimes \mathrm{T}^{\ell}\left(\Omega_{X}^{1}\right)\right)$.

Theorem 4.8. When $K_{X} \cdot \mathrm{H}^{n-1} \geq 0$, we have, for any $\mathcal{E} \subset F_{*} W$,

$$
\mu\left(F_{*} W\right)-\mu(\mathcal{E}) \geq-\frac{\mathrm{I}(W, X)}{p} .
$$

In particular, if $W \otimes \mathrm{T}^{\ell}\left(\Omega_{X}^{1}\right), 0 \leq \ell \leq n(p-1)$, are semistable, then $F_{*} W$ is semistable. Moreover, if $K_{X} \cdot \mathrm{H}^{n-1}>0$, the stability of the bundles $W \otimes \mathrm{T}^{\ell}\left(\Omega_{X}^{1}\right), 0 \leq \ell \leq n(p-1)$, implies the stability of $F_{*} W$.

Proof. Since $K_{X} \cdot \mathrm{H}^{n-1} \geq 0$, by the inequality (4.9) in Lemma 4.4 (see also the notation in (4.8) and the lemma), it is enough to show

$$
\sum_{\ell=0}^{m}\left(\frac{n(p-1)}{2}-\ell\right) r_{\ell} \geq 0 .
$$


If $m \leq \frac{n(p-1)}{2}$, it is clear. If $m>\frac{n(p-1)}{2}$, then we have

$$
\begin{aligned}
& \sum_{\ell=0}^{m}\left(\frac{n(p-1)}{2}-\ell\right) r_{\ell}=\sum_{\ell=m+1}^{n(p-1)}\left(\ell-\frac{n(p-1)}{2}\right) r_{n(p-1)-\ell} \\
& +\sum_{\ell>\frac{n(p-1)}{2}}^{m}\left(\ell-\frac{n(p-1)}{2}\right)\left(r_{n(p-1)-\ell}-r_{\ell}\right) .
\end{aligned}
$$

We will use Proposition 4.7 to show that

$$
r_{\ell} \leq r_{n(p-1)-\ell} \quad \text { when } \frac{n(p-1)}{2} \leq \ell \leq n(p-1)
$$

It is clearly a local problem, we can consider all of the torsion free sheaves as vector spaces over the function field $K=k(X)$ of $X$. Without loss of generality, we assume $\operatorname{rk}(W)=1$. Then, from the discussions in Section 3, we know that $V_{\ell} / V_{\ell+1} \cong \mathrm{T}^{\ell}\left(\Omega_{X}^{1}\right)(0 \leq \ell \leq n(p-1))$ are precisely isomorphic to $R^{\ell}(0 \leq \ell \leq n(p-1))$ in Proposition 4.7 . Since the morphisms $V_{\ell} / V_{\ell+1} \stackrel{\nabla}{\rightarrow}\left(V_{\ell-1} / V_{\ell}\right) \otimes \Omega_{X}^{1}$ induce morphisms $\mathcal{F}_{\ell} \stackrel{\nabla}{\rightarrow} \mathcal{F}_{\ell-1} \otimes \Omega_{X}^{1}$, by the formula (3.6), we have

$$
\mathrm{D}_{2 \ell-n(p-1)} \cdot \mathcal{F}_{\ell} \subset \mathcal{F}_{n(p-1)-\ell} .
$$

Then, by Proposition 4.7, $r_{\ell}=\operatorname{dim}\left(\mathcal{F}_{\ell}\right) \leq \operatorname{dim} \mathbb{L}\left(\mathrm{D}_{2 \ell-n(p-1)} \cdot \mathcal{F}_{\ell}\right)$, we have $r_{\ell} \leq r_{n(p-1)-\ell}$, thus (4.15).

If the bundles $W \otimes \mathrm{T}^{\ell}\left(\Omega_{X}^{1}\right)(0 \leq \ell \leq n(p-1))$ are stable, then

$$
\mu\left(F_{*} W\right)-\mu(\mathcal{E}) \geq 0
$$

It becomes equality if and only if inequalities (4.10) become equalities and $\sum_{\ell=0}^{m}\left(\frac{n(p-1)}{2}-\ell\right) r_{\ell}=0$. Thus $m>\frac{n(p-1)}{2}$ and each term in (4.16) must be zero (since $K_{X} \cdot \mathrm{H}^{n-1}>0$ ), which forces $m=n(p-1)$. Then the fact that inequalities (4.10) become equalities implies $\mathcal{E}=F_{*} W$.

Corollary 4.9. Let $X$ be a smooth projective variety of $\operatorname{dim}(X)=n$, whose canonical divisor $K_{X}$ satisfies $K_{X} \cdot \mathrm{H}^{n-1} \geq 0$. Then

$$
\mathrm{I}\left(F_{*} W\right) \leq p^{n-1} \operatorname{rk}(W) \mathrm{I}(W, X) .
$$

Proof. It is just Theorem 4.8 plus the following trivial remark: For any vector bundle $E$, if there is a constant $\lambda$ satisfying $\mu\left(E^{\prime}\right)-\mu(E) \leq \lambda$ for any $E^{\prime} \subset E$. Then $\mathrm{I}(E) \leq \operatorname{rk}(E) \lambda$. 


\section{REFERENCES}

[1] Biswas, I., Holla, Y. : Comparison of fundamental group schemes of a projective variety and an ample hypersurface, arXiv: math.AG./0603299v1, 13 March, (2006).

[2] Biswas, I., Holla, Y. : Comparison of fundamental group schemes of a projective variety and an ample hypersurface, J. Algebraic Geom. 16 (2007), 547-597.

[3] Doty, S., Walker, G.: Truncated symmetric powers and modular representations of $\mathrm{GL}_{n}$, Math. Proc. Camb. Phil. Soc. 119 (1996), 231-242.

[4] Joshi, K., Ramanan, S., Xia, E.Z., J.-K., Yu: On vector bundles destabilized by Frobenius pull-back, arXiv: math.AG./0208096 v1, 13 Aug. (2002). Compositio Math. 142 (2006), 616-630.

[5] Katz, N.: Nilpotent connection and the monodromy theorem: Application of a result of Turrittin, I.H.E.S. Publ. Math. 39 (1970), 175-232

[6] Lange, H., Pauly, C. : On Frobenius-destabilized rank two vector bundles over curves, arXiv: math.AG./0309456 v2, 6 Oct. (2005).

[7] Mehta, V., Pauly, C. : Semistability of Frobenius direct images over curves, arXiv: math.AG./0607565 v1, 22 July, (2006).

[8] Sun, X. : Stability of direct images under Frobenius morphisms, arXiv: math.AG./0608043 v1, 2 Aug. (2006).

Academy of Mathematics and Systems Science, Chinese Academy of Science, BeiJing, P. R. of China

E-mail address: xsun@math.ac.cn 